\title{
A SHORT PROOF OF A THEOREM OF BIRKHOFF
}

\author{
G. GRÄTZER, E. T. SCHMIDT, AND D. WANG
}

\begin{abstract}
This paper gives a new proof of a theorem of G. Birkhoff: Every group $\mathfrak{G}$ can be represented as the automorphism group of a distributive lattice $D$; if $\mathfrak{G}$ is finite, $D$ can be chosen to be finite. The new proof is short, and it is easily visualized.
\end{abstract}

\section{INTRODUCTION}

In [1], G. Birkhoff proved the following result:

Theorem. Every group $\mathfrak{G}$ can be represented as the automorphism group of a distributive lattice $D$; if $\mathfrak{G}$ is finite, then $D$ can be chosen to be finite.

See also the references for alternative proofs. In this note, we present a new proof of this result which may be the simplest and most direct of all the proofs.

\section{THE PROOF}

Let $\mathfrak{G}$ be the given group defined on the set $G=\left\{g_{\gamma} \mid \gamma<\alpha\right\}$ with $g_{0}=1$; we assume that $|G|>1$. We view ordinals as well-ordered chains, so

$$
\gamma \leq \delta<\alpha \text { and } \gamma \cong \delta \text { imply that } \gamma=\delta \text {. }
$$

For every $x, y \in G$ with $y \neq 1$ (equivalently, with $x \neq y x$ ), we construct the poset $P(x, y)$ of Figure 1 , defined on the set $\{x, y x,\langle x, y\rangle\} \cup(\psi \times\langle x, y\rangle)$, where $y=g_{\psi}, \psi<\alpha$; we partially order this set by

$$
\begin{gathered}
x<\langle 0,\langle x, y\rangle\rangle\langle\langle 1,\langle x, y\rangle\rangle<\cdots<\langle\gamma,\langle x, y\rangle\rangle<\ldots, \quad \gamma<\psi ; \\
y x<\langle x, y\rangle<\langle 0,\langle x, y\rangle\rangle .
\end{gathered}
$$

The two minimal elements of $P(x, y)$ are $x$ and $y x$, both in $G$.

Let $P=\bigcup(P(x, y) \mid x, y \in G, y \neq 1)$ be partially ordered by $a<b$ in $P$ iff $a<b$ in some $P(x, y)$. It is sufficient to prove that Aut $P \cong \mathfrak{G}$. Indeed, Aut $P \cong$ Aut $L$, where $L$ is the distributive lattice completely freely generated by $P$; moreover, if $G$ is finite, then $P$ and $L$ are both finite.

To prove that Aut $P \cong \mathfrak{G}$, let $\Sigma$ be an automorphism of $P$. Since $G$ is the set of minimal elements of $P$, so $\Sigma$ permutes $G$. Let $a=1 \Sigma$ and let $b \in G$. We want to show that $b \Sigma=b a$.

If $b=1$, this holds by the definition of $a$. So let $b \neq 1$. Let $b=g_{\beta}$ with $\beta<\alpha$. Then the poset $P(1, b)$, with minimal elements 1 and $b$, is defined (since $1 \neq b=b 1$ ).

Date: September 30, 1995.

1991 Mathematics Subject Classification. Primary:06D05, Secondary:06A06.

Key words and phrases. group, poset, automorphism group, distributive lattice.

The research of the first author was supported by the NSERC of Canada.

The research of the second author was supported by the Hungarian National Foundation for Scientific Research, under Grant No. 1903. 


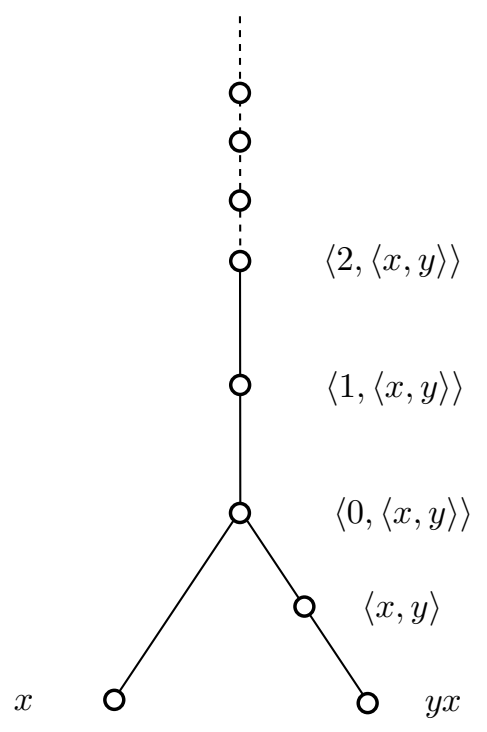

Figure 1 . The poset $P(x, y)$

Since $b \neq 1$, also $b \Sigma \neq a(=1 \Sigma)$ and so $b \Sigma=u a$, for some $u \in G$ with $u \neq 1$. Therefore, $P(a, u)$ with minimal elements $a=1 \Sigma$ and $u a=b \Sigma$, is defined.

Thus $\Sigma$ takes the minimal elements of $P(1, b)$ into the minimal elements of $P(a, u)$, hence it must take all of $P(1, b)$ to $P(a, u)$, so $P(1, b) \cong P(a, u)$. Thus the top chain of $P(a, u)$ is the same as the top chain of $P(1, b)$, that is, $\beta$, and so $u=b$, proving that $b \Sigma=b a$.

For every $a \in G$, define $\Sigma_{a}$ by $b \Sigma_{a}=b a$. Then we have just proved that every automorphism of $P$ restricted to $G$ is of this form; the converse is trivial. This completes the proof of the claim and of the Theorem.

\section{REFERENCES}

[1] G. Birkhoff, On the groups of automorphisms (Spanish), Revista Unió Mat. Argentina 11 (1946), 155-157.

[2] R. Frucht, Herstellung von Graphen mit vorgegebener abstrakter Gruppe, Compos. Math. 6 (1938), 239-250.

[3] Lattices with a given group of automorphisms, Canad. J. Math. 2 (1950), 417-419.

[4] G. Grätzer, General Lattice Theory, Pure and Applied Mathematics 75, Academic Press, Inc. (Harcourt Brace Jovanovich, Publishers), New York-London; Lehrbücher und Monographien aus dem Gebiete der Exakten Wissenschaften, Mathematische Reihe, Band 52. Birkhäuser Verlag, Basel-Stuttgart; Akademie Verlag, Berlin, 1978. xiii+381 pp.

[5] G. Grätzer and H. Lakser, Homomorphisms of distributive lattices as restrictions of congruences. II. Planarity and automorphisms, Canadian J. Math. 46 (1) (1994), 3-54.

[6] G. Grätzer, H. Lakser, and E. T. Schmidt, On a result of Birkhoff, Period. Math. Hungar. 30 (1995), 183-188. 
Defartment of Mathematics and Astronomy, University of Manitoba, Winnif eg, ManITOBA, CANADA R3T 2N2

E-mail address: George_Gratzer@umanitoba.ca

Department of Mathematics, Transport Engineering Faculty, Technical University of Budafest, Müegyetem Rkp. 9, 1111 Budapest, Hungary

E-mail address: schmidt@euromath.vma.bme.hu

Department of Mathematics and Astronomy, University of Manitoba, Winnifeg, ManITOBA, CANADA R3T 2N2

E-mail address: Dabin_hang@umanitoba.ca 